\title{
Understanding PD-L1 Testing in Breast Cancer: A Practical Approach
}

\author{
Ramona Erber Arndt Hartmann \\ Institute of Pathology, University Hospital Erlangen, Friedrich-Alexander University Erlangen-Nürnberg (FAU), \\ Comprehensive Cancer Center Erlangen-EMN, Erlangen, Germany
}

\author{
Keywords \\ Programmed death-1 protein (PD-1) · PD-L1 · Checkpoint \\ therapy · Immunotherapy · Breast cancer · Triple negative \\ breast cancer $\cdot$ TNBC $\cdot$ Immune cells
}

\begin{abstract}
Background: Immune checkpoint inhibitors (ICI) have changed therapy strategies for cancer patients tremendously. Some approved ICl acquire testing of PD-L1 expression on tumor and/or immune cells. However, since PD-L1 testing is a comprehensive issue with various assays, antibody clones, scoring methods, and cut-offs, we aimed to summarize the recommendations and technical and histopathological issues of diagnostic PD-L1 assessment with an emphasis on invasive breast cancer (IBC). Summary: Besides other (pre) analytical considerations, selecting the most adequate PDL1 immunohistochemical assay/antibody clone is important. In-house assay validation, prediagnostic training, and internal and external quality assurance should be implemented. The current most relevant PD-L1 assays and scores will be explained in this review. Moreover, recommendations for PD-L1 testing in IBC are outlined. Key Messages: Atezolizumab plus nab-paclitaxel therapy is approved for adult patients with locally advanced or metastatic triple negative breast cancer (mTNBC), if the tumor-associated immune cells express PD-L1. - This PD-L1 immune cell positivity is defined as an immune cell (IC) score, which refers to the area occupied by PD-L1 positive immune cells (lymphocytes, dendritic cells, macrophages, and granulocytes) as a percentage of the whole tumor area. The cut-off is an IC score $\geq 1 \%$. In the approval study for atezolizumab in mTNBC, IC
\end{abstract}

score was assessed using the Ventana PD-L1 SP142 assay. Other assays or laboratory developed tests may be used depending on country-specific drug approvals. However, harmonization studies have to show whether other PD-L1 tests are reliable and of clinical value to predict the response of breast cancer patients to $\mathrm{ICl}$.

(c) 2020 S. Karger AG, Basel

\section{Introduction}

Therapeutic management of invasive breast cancer (IBC) is guided by multiple prognostic and predictive clinical and pathological features. Deciding for or against endocrine therapy, chemotherapy, and/or targeted therapy (e.g., anti-HER2 therapy) is, amongst other factors, dependent on the intrinsic IBC subtype. These subtypes include luminal tumors, HER2-enriched (HER2+) IBC, and triple-negative breast cancer (TNBC), and they are both prognostic and predictive [1-7]. Besides systemic therapy possibilities mentioned above, multiple innovative drugs (e.g., CDK4/6 inhibitors) that target specific oncogenes have been reported to improve the outcome of breast cancer patients [8]. In addition, immunotherapeutic strategies including immune checkpoint inhibitors (ICI) have proven effective in many neoplastic entities and have changed the field of oncology tremendously. Targeting the programmed death-1 protein (PD-1) and its ligand, PD-L1, is currently the recommended standard therapy in many advanced or metastatic tumors (e.g., non-small-cell lung cancer, urothelial carcinoma, Merkel cell carcinoma, classic Hodgkin lymphoma, head and 
neck and cutaneous squamous-cell carcinoma, renal cell carcinoma) [9-11]. In metastasized (m)TNBC that show immunohistochemical PD-L1 expression on tumor-associated immune cells, the addition of anti-PD-L1 therapy (atezolizumab) to nab-paclitaxel has been shown to be superior to nab-paclitaxel monotherapy regarding survival $[12,13]$. For some combinations of ICI, tumor entity, and clinical setting (tumor stage and line of therapy), drug approval requirements demand the detection of $\mathrm{PD}$ L1 expression on tumor cells and/or immune cells [11]. However, PD-L1 testing is a comprehensive issue.

We aimed here to summarize the recommendations and issues involved in diagnostic PD-L1 detection with an emphasis on IBC. We have not made definitive conclusions, however, because this field is developing very fast and new insights into immune checkpoint inhibition are constantly being made.

\section{PD-L1 Testing in Breast Cancer}

\section{The PD-1/PD-L1 Axis: An Immune Checkpoint}

Briefly, PD-1 (CD279) is an inhibitory coreceptor which is expressed on the surface of T lymphocytic cells. The activation of this coreceptor through binding of the ligand PD-L1 (B7-H1; CD274), which can be expressed on some immune cells, leads to the inhibition of the T cell response, to self-tolerance and immune tolerance, i.e., physiologically, the PD-1/PD-L1 axis protects us from excessive immune responses and autoimmune reactions [14]. Many solid, and also some hematopoietic, neoplasms express $\mathrm{PD}-\mathrm{L} 1$ to inactivate the $\mathrm{T}$ cell response, thereby bypassing this immune checkpoint and the response of our immune system to neoplastic cells $[15,16]$. This mechanism is also known as immune escape or local suppression of the immune system. Therapeutic monoclonal antibodies, so-called ICI, against PD-1 or PD-L1, can, however, suspend this inhibitory effect of the PD-1/ PD-L1 axis with T cells and the endogenous antitumoral immune response is thus reactivated $[9,10,14,17]$.

\section{PD-L1 Testing in General}

In routine diagnostics, $\mathrm{PD}-\mathrm{L} 1$ expression is measured using immunohistochemistry (IHC). However, various commercially available assays and numerous antibody clones for PD-L1 detection as well as multiple expression scores and cut-offs exist. Since these different IHC staining and scoring methods have each been specifically used to evaluate the clinical efficacy of a particular ICI in clinical studies, (i) the approval of ICI is often linked to a certain PD-L1 IHC assay (given that an assessment of the PD-L1 status is required), and (ii) direct comparison of the predictive value of various IHC antibody clones and PD-L1 scores/cut-offs is hampered [18]. Harmonization studies have been performed to address this issue and investigate the most reliable assays and antibody clones [1923]. However, there is still a need to compare the different staining and counting methods and their predictive values for ICI response, especially in breast cancer [18].

\section{PD-L1 IHC Assays and Antibody Clones}

The commercially available diagnostic PD-L1 assays used in clinical trials, including specific antibody clones, are listed in Table 1. As already mentioned, using a particular score and cut-off, each has been specifically implemented in these studies in relation to the clinical testing of 1 specific ICI. Furthermore, they have been validated with specific platforms. Hence, laboratories would have to provide several assays, antibody clones, platforms, and validated in-house protocols for each assay if every ICI were associated with a single companion diagnostic test. US FDA approval of some ICI are indeed associated with a specific companion diagnostic test [24]. This is not current practice in the European Union. Hence, many European pathological laboratories have established laboratory-developed tests (LDTs). Besides those in common use, other PD-L1 antibody clones are available, e.g., E1L3N (Cell Signalling), CAL10 (Zytomed), QR1 (Quartett), and ZR3 (Cell Mark) [18].

\section{IHC Staining and Reporting}

Important preanalytical and analytical considerations have to be implemented for adequate PD-L1 testing. When requesting PD-L1 IHC, the clinician should provide the pathologist with information about the intended ICI therapy, as the clinical trials that led to the approval of the ICI are each associated with a specific companion in vitro diagnostic (CIVD) IHC assay and a particular score.

The issue as to which tissue should be used is further discussed in detail for IBC in this review. In general, however, biopsies or surgical specimens should be fixated in neutral-buffered formalin for 6-72 $\mathrm{h}$, depending on the sample size, and then processed further according to the usual practice. It is recommended that whole-slide sections approximately $4-\mu \mathrm{m}$-thick are cut from representative areas of the formalin-fixed and paraffin-embedded (FFPE) tumor tissue shortly before IHC, or else stored no longer than a few weeks at room temperature. Over time, perfect staining results cannot be guaranteed due to the possibility of tissue degradation and antigen loss on archived slides. The PD-L1 IHC staining protocol should be according to the assay manufacturer's recommendations and established in-house; in Germany, this is according to the general recommendation (e.g., in guidelines), and for accredited institutions, according to the requirements of the national accreditation body of the Federal Republic of Germany (DAkkS) before the first diagnostic use. 
The testing should contain an external positive control. FFPE tonsil tissue is a cost-effective alternative to PD-L1-positive cell lines. The tonsil parenchyma contains physiologically both moderately-to-strongly PD-L1positive immune cells (lymphocytes and monocytes) dispersed in the paracortical region, or in clusters within the germinal centers, and strongly positive epithelial cells of the tonsil crypts, but the surface epithelium shows no PDL1 expression. Therefore, tonsil tissue can be used as staining control of both epithelial cells and immune cells (Fig. 1). If the reticulated crypt epithelium is positive but immune cells do not show any PD-L1 positivity, the staining procedure should be repeated. Moreover, a negative control, and, if applicable, a control that shows PD-L1 expression near the cut-off, are also recommended [16, $18,25-27]$.

After reviewing the tumor on a corresponding hematoxylin and eosin (H\&E) slide, the PD-L1 IHC slide is evaluated semiquantitatively by the pathologist. A neoplastic cell is counted as PD-L1-positive if there is a membranous staining, irrespective of staining intensity and whether the membrane depicts complete or partial PDL1 positivity. If there is cytoplasmic but no membranous staining, a tumor cell is considered PD-L1-negative. For immune cells, however, either granular cytoplasmic or (incomplete) membranous staining suffices for a positive count (Fig. 2) [16, 19, 23]. The varying scoring methods are explained in the section below. For pathologists that assess PD-L1 staining, training in PD-L1 assessment and annual internal and external quality assurance (EQA) is recommended $[18,26]$.

The pathologist's report should contain information about the antibody clone used for IHC, the percentage of PD-L1-positive immune cells, and/or tumor cells and the score(s) relevant to the therapeutic setting of the respective tumor and approved drug. The staining assay and platform used can be mentioned additionally $[18,20,27$, 28].

\section{Relevant PD-L1 Scores}

Tumor Cell Score. The tumor cell score is defined as a percentage of the area covered by PD-L1-positive tumor cells in relation to the whole tumor area [27].

Tumor Proportion Score. The tumor proportion score defines the ratio of PD-L1-positive tumor cells, relative to all vital tumor cells, multiplied by $100 \%[29,30]$.

Immune Cell Score. All immune cells that are located intratumorally or in a small peritumoral stromal rim are taken into account when calculating the IC score. The method evaluates the percentage of the area occupied by all PD-L1-positive immune cells (lymphocytes, dendritic cells, macrophages, and granulocytes) relative to the whole tumor area (neoplastic cells and tumor stroma; Fig. 2, 3). The IC can be subclassified as follows:

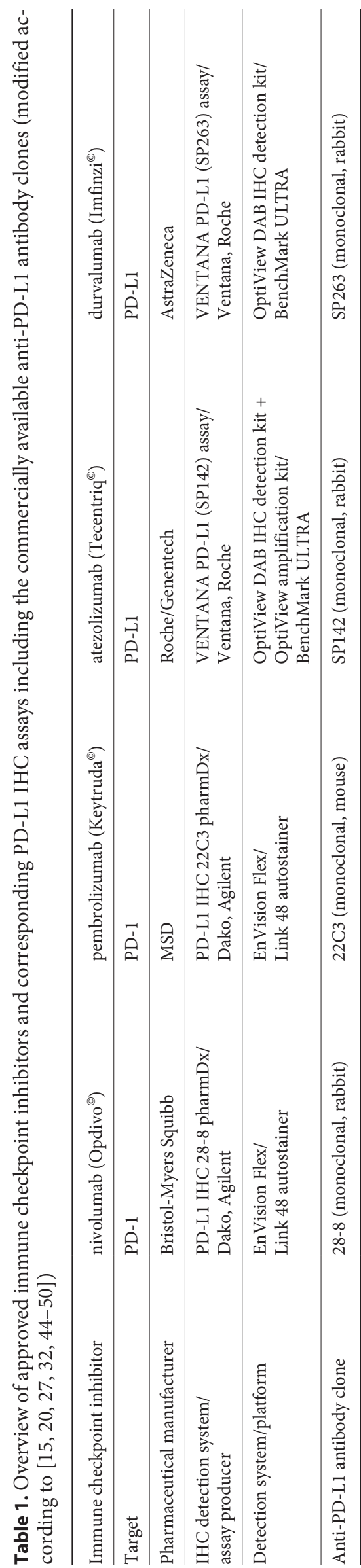




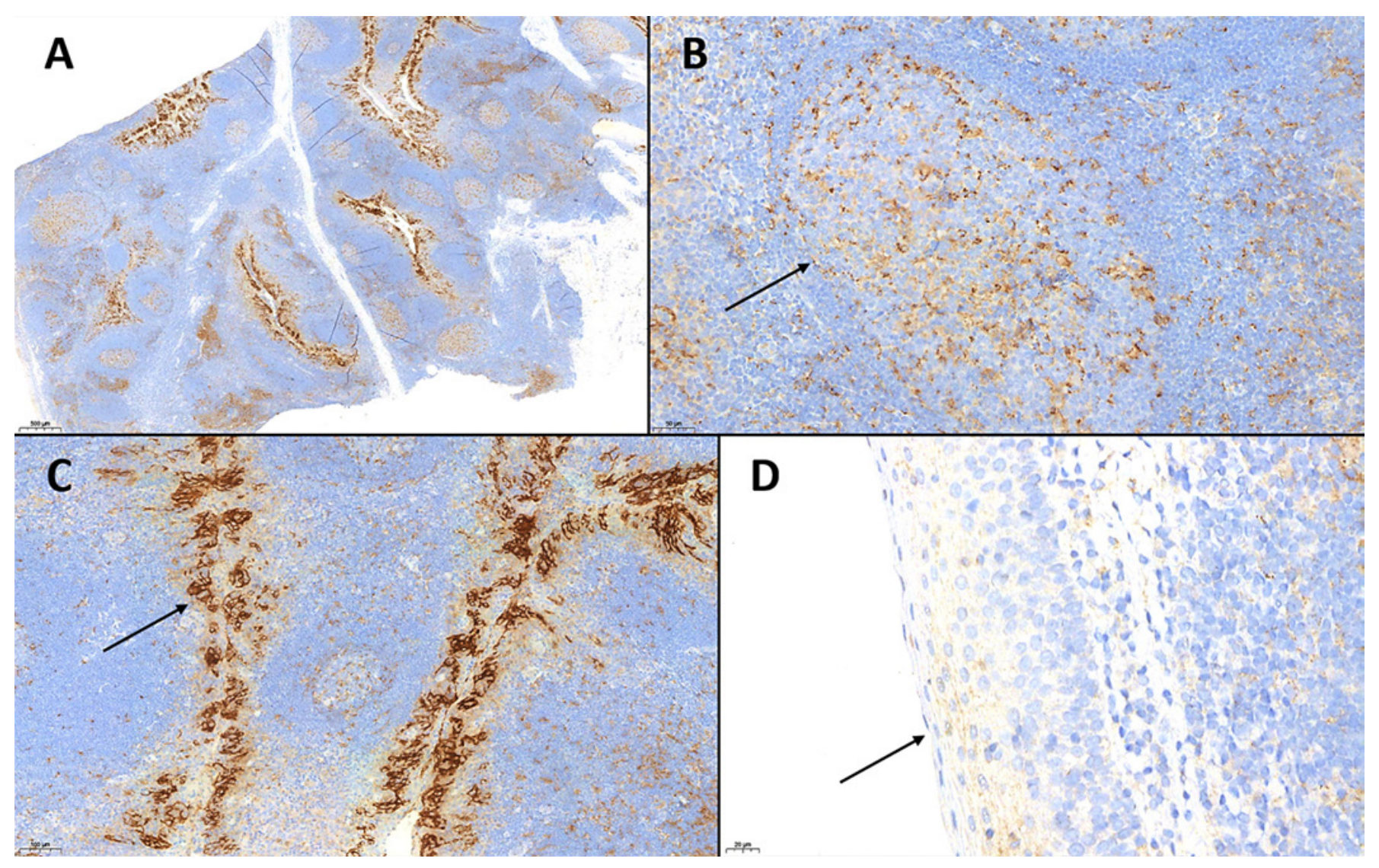

Fig. 1. A PD-L1 IHC of a tonsil used as positive control. Note the tonsil parenchyma containing physiologically both PD-L1-positive immune cells (lymphocytes and monocytes), dispersed in the paracortical region (B) or in clusters within the germinal centers (B, arrow), and positive epithelial cells of the tonsil crypts (C). D The surface epithelium does not show any PD-L1 expression (each PD-L1 IHC, clone SP263., A ×20. B ×200. C ×100. D ×400.

- IC0: $<1 \%$

- IC1: $\geq 1$ to $<5 \%$

- IC2: 5 to $<10 \%$

- IC3: $\geq 10 \%[12,15]$.

Combined Positive Score. This score involves both tumor cells and intratumoral immune cells or those in a narrow rim around the tumor (lymphocytes and macrophages; neutrophil granulocytes do not count). The number of PD-L1-positive tumor cells and PD-L1-positive immune cells is summarized, relative to the number of all vital tumor cells, and then multiplied by 100 . The combined positive score (CPS) is stated without any units. Although values $>100$ could theoretically be achieved, the maximum CPS is defined as 100 [27, 29, 31-32].

\section{PD-L1 Testing in Breast Cancer}

The randomized phase III IMpassion 130 study investigated the benefit of adding atezolizumab to nab-paclitaxel for patients with locally advanced TNBC or mTNBC without prior treatment of the metastasized disease. Since this combination of ICI plus chemotherapy showed improved survival of patients, both the FDA and the Euro- pean Medicines Agency (EMA) approved atezolizumab for treatment in the aforementioned setting. This admission, however, is linked to the detection of PD-L1-positive immune cells with an IC score of at least $1 \%[12,33$, 34]. In the IMpassion 130 trial, Ventana PD-L1 SP142 was used, and for the primary efficacy end points, the intention-to-treat cohort as well as the PD-L1-positive subgroup (IC score $\geq 1 \%$ ) were analyzed. Therefore, the FDA has approved Ventana PD-L1 SP142 as a CIVD test [24]. A post hoc study of this patient cohort aimed to harmonize PD-L1 assays (the Ventana SP142 and SP263 IHC assays and the Dako 22C3 IHC assay). Cut-offs for 22C3 and SP263 were derived by modeling them as those that maximize analytical agreement. The results showed that 22C3 and SP263 identified other PD-L1-positive populations differing from SP142 results. However, as highlighted by the authors, these data should be interpreted carefully since cut-offs were based on mathematical modeling [35]. Further studies, testing the efficacy of varying ICI for breast cancer patients in molecular IBC subgroups and various settings, have been reported [36-38] or are still ongoing. However, there is currently no further ap- 


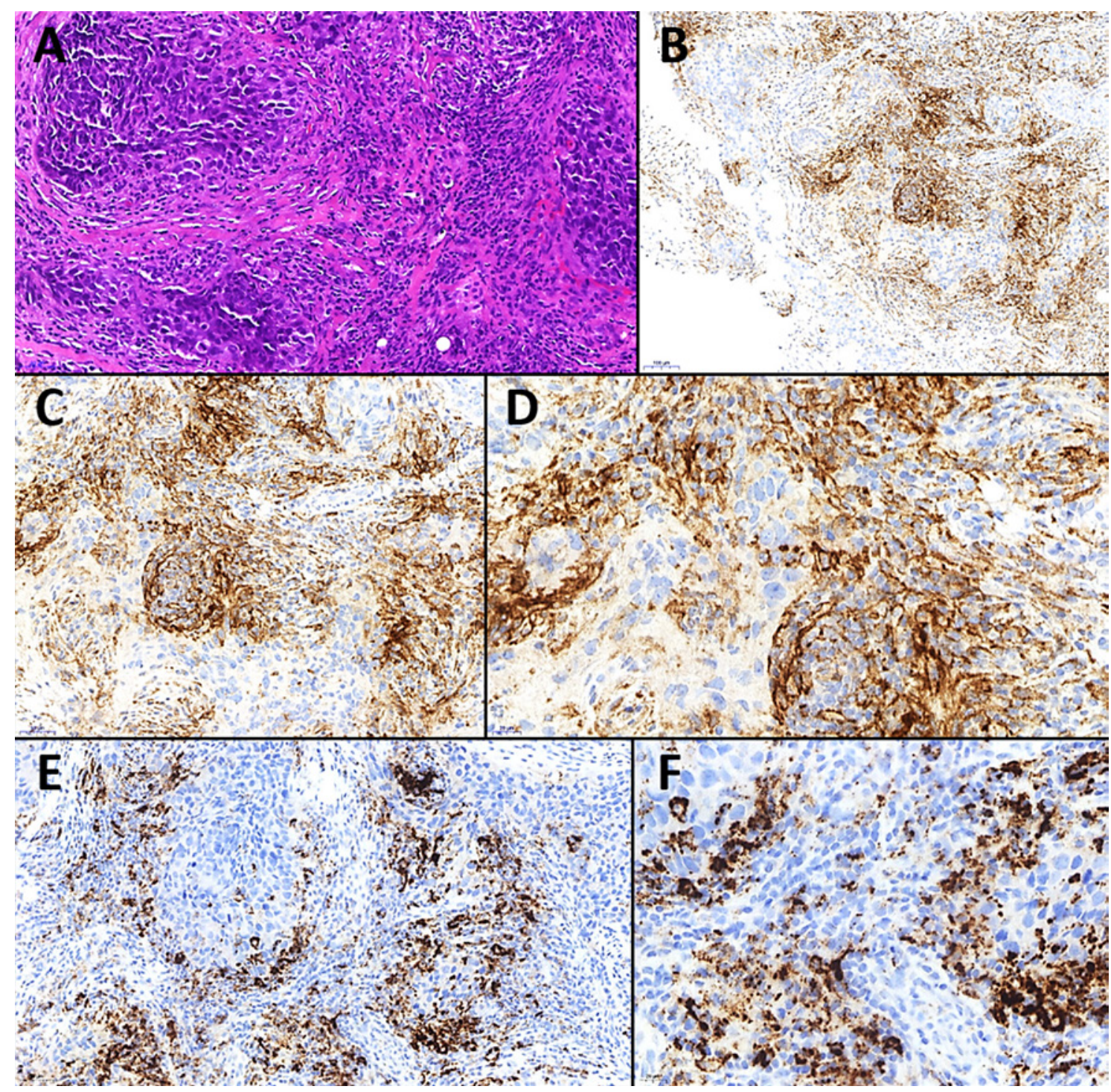

Fig. 2. Triple-negative breast cancer with positive PD-L1 expression. A The histology of the invasive breast cancer. Note the prominent stromal inflammatory infiltrate with abundant tumor-infiltrating lymphocytes (TILs). H\&E. $\times 200$. For both PD-L1 assays, SP263 $(\mathbf{B} \times 100, \mathbf{C} \times 200, \mathbf{D} \times 400)$ and SP142 $(\mathbf{E} \times 200, \mathbf{F} \times 400)($ Staining, courtesy of Prof. Wilko Weichert), PD-L1 IHC showed positivity of TILs (immune cell [IC] score $>1 \%$ ). B-D Using SP263, few tumor cells showed membranous PD-L1 staining. E, F In contrast, the SP142 assay did not. However, this phenomenon is known and might be due to the fact that the SP142 assay was established to detect immune cells with high sensitivity, but not epithelial/tumor cells.

proval of any ICI that is linked to the detection of PD-L1 in IBC.

In conclusion, the following workflow has to be fulfilled if an adult patient with unresectable locally advanced TNBC or mTNBC is diagnosed and atezolizumab plus nab-paclitaxel therapy is intended. The oncologist orders PD-L1 testing with the information that this ICI therapy is planned. Besides the considerations already mentioned in the section "IHC Staining and Reporting" and the relevant scoring method, i.e., IC score, that both apply also for PD-L1 testing in IBC, some issues have to be highlighted for the specific setting of atezolizumab therapy in mTNBC (modified according to $[18,39,40]$ ).

- Appropriate FFPE tissue:

- Both archived tissue blocks of the primary IBC as well as material from the metastasis is adequate. A word of caution: to date, there is some (but insufficient) data about PD-L1 inter-tumoral heterogeneity, and it is unclear whether (I) both the primary tumor and metastasis, should be tested, (II) in which sequence the available tissue should be analyzed, and (III) which procedure is most predictive for the efficacy of ICI therapy 



Fig. 3. Evaluation of the IC score (\%) using PD-L1 IHC. A After assessment of the whole tumor area, PD-L1-positive immune cells (IC) are reviewed. B-D All cytoplasmicly and/or membranously positively stained IC located intratumorally or in a small peritumoral stromal rim are taken into account. Switching back to the overview $(\mathbf{A})$, the IC score is assessed as the percentage of the area occupied by all PD-L1-positive IC (lymphocytes, dendritic cells, macrophages, and granulocytes) relative to the whole tumor area (neoplastic epithelial cells and tumor stroma). In this case, the IC score is $5 \%$. Note the membranously PD-L1-positive tumor cells; these are not taken into account for IC score (each PD-L1 IHC clone SP263, A $\times 11, \mathbf{B} \times 20, \mathbf{C} \times 100, \mathbf{D} \times 200$ ).
$[41,42]$. In general, however, the current recommendation is to test the most recent tissue.

- Biopsies, excisional, or surgical specimens can be used.

- According to the manufacturer's statements, however, the Ventana PD-L1 SP142 assay has not been validated for cytological material or decalcified bone metastases.

- PD-L1 IHC assay:

- Depending on the respective drug approval in each country, the Ventana PD-L1 SP142 assay has to be applied (e.g., US FDA) or is at least recommended. However, other validated and in-house established assays or LDTs may be used in some other countries; in some European countries including Germany, EMA approval of the drug is currently not bound to testing with a specific immunohistochemical antibody. Moreover, it has to be mentioned that the cost of commercially available PD-L1 assays can be very high, potentially leading to a gap between costs for the pathologists and reimbursement. In Germany, the 10-fold greater cost of the commercial CE-certified tests has led to the frequent use of LDTs in many pathology laboratories. The results of first harmonization trials are discussed below.

- Assessment of the IC score for TNBC by the pathologist:

- Reviewing the H\&E slide, at least 50 (-100) vital tumor cells and tumor-associated stroma should be present, and the whole tumor area (excluding necrotic areas) has to be evaluated. Moreover, the presence or absence of and the distribution of tumor-associated immune cells must be recognized.

- Controls have to show adequate staining results. If this is not the case, the IHC staining procedure has to be repeated.

- Assessing the PD-L1 IHC expression of the TNBC slide, immune cells (lymphocytes, dendritic cells, macrophages, and granulocytes) can be found in clusters or singly dispersed. Any cytoplasmic or membranous PD-L1 positivity of the immune cells counts. The area 


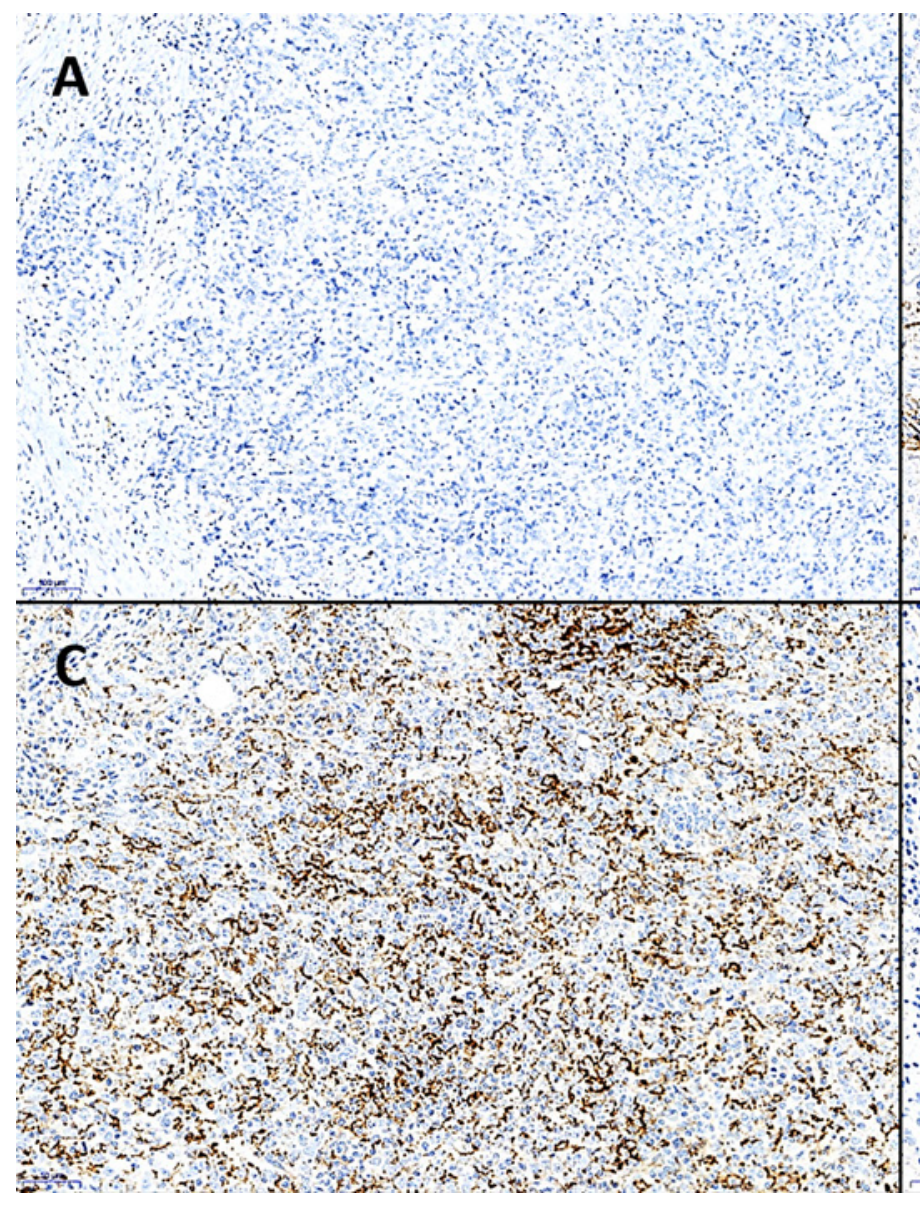

Fig. 4. Varying IC score (\%) in triple-negative breast cancer. For assessment, PD-L1 IHC was used (PD-L1 clone SP263). A The first case did not show any PD-L1 positivity and was therefore considered PD-L1-negative (IC score $=0 \%) . \times 100$. B, C Both samples display an IC score $>1 \%$ with $\mathbf{C}$ showing many more positive tu-

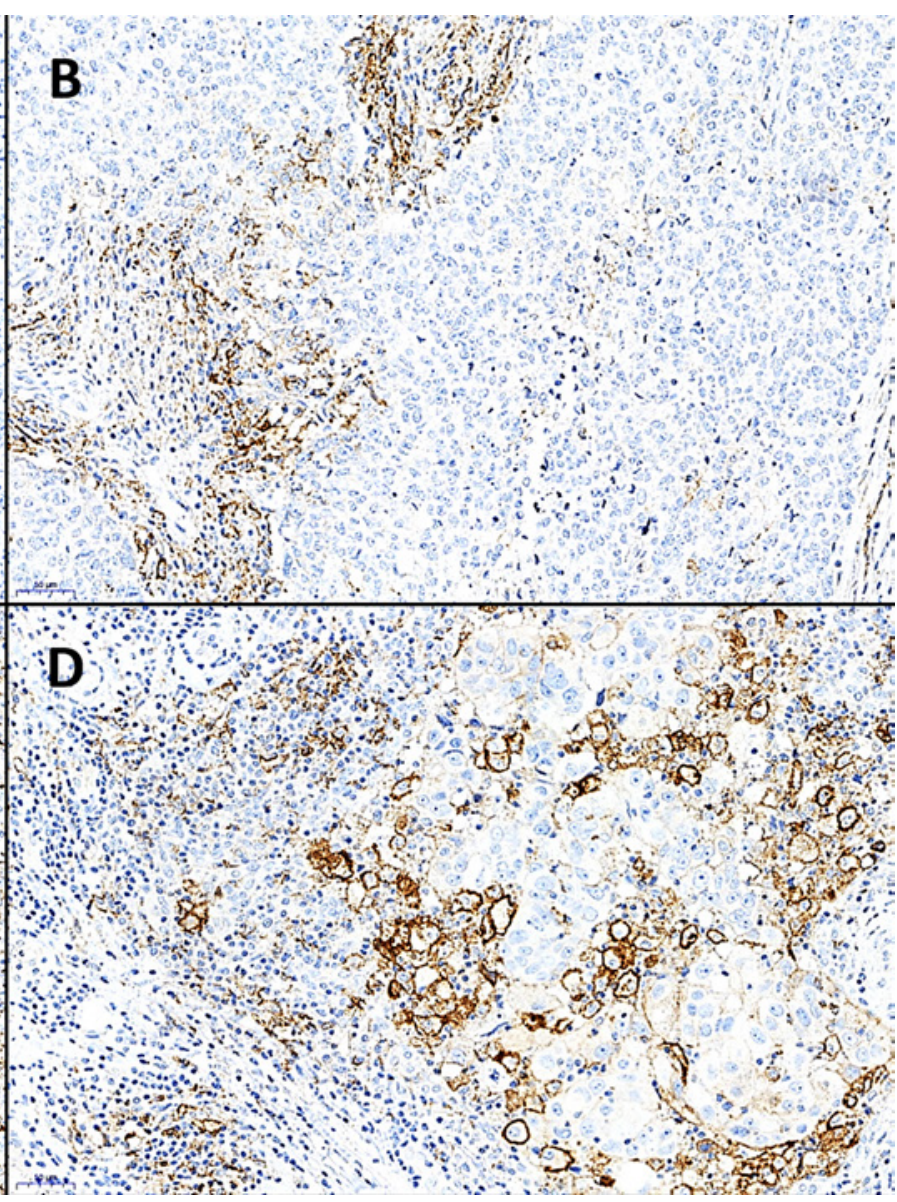

mor-associated immune cells $($ IC score $=25 \%)$ than $\mathbf{B}($ IC score $=$ $4.5 \%)$. D The IC score is $>1 \%$; however, note the strongly membranously PD-L1-positive tumor cells which are clearly larger than the mostly granular stained immune cells; the tumor cells must not be considered for the IC score (IC score $=5 \%)$. B-D $\times 200$. that is occupied by all PD-L1-positive immune cells is recorded and then related to the whole vital tumor area (\%). For instance, the area of PD-L1-positive immune cells comprises one-tenth of the tumor area so the IC score is 10\%. In Figure 4, 1 negative and 3 PD-L1-positive TNBC samples are depicted.

- Staining artefacts, necrosis, or intravascular immune cells have to be excluded from the evaluation.

- PD-L1-positive tumor cells are not required for the IC score. Since the results of ongoing clinical trials will probably change the current situation regarding ICI approvals for IBC, oncologists and pathologists have to be in a lively exchange as to which PD-L1 assay/LDT and which PD-L1 score(s) are required.

- A practical tip for the pathologists (applicable to all solid tumor entities that have to be tested for PD-L1) is: in the case of unknown history of the patient and unknown prior or planned therapy (study enrollment?), report the following:
1. the IHC assay/LDT and antibody clone used; the platform may be added

2. the percentage of PD-L1-positive immune cells and tumor cells and the common scores with emphasis on the currently relevant/approved scoring method (i.e., for TNBC, the IC score)

3. if there is uncertainty about the relevant score(s), call the corresponding oncologist.

- Quality assurance (QA):

- It is strongly recommended that pathologists are trained in PD-L1 assessment prior to implementing it in routine diagnostics.

- For internal QA, rates of PD-L1-positive cases should be correlated with the results of clinical studies. Prevalence of TNBC with any IC score $\geq 1 \%$ fluctuates between 32 and $86 \%$, depending on the assay used (e.g., $41 \%$ for SP142). The review by Gonzalez-Ericsson et al. [18] provides a comprehensive overview of published prevalences. 
- Participation in external QA is highly recommended. Organizations like the Nordic Immunohistochemical Quality Control (NordiQC) and the German Quality Assurance Initiative Pathology (QuIP) offer such round-robin tests.

- Harmonization of PD-L1 IHC assays in IBC:

- In a multicenter assay comparison study, moderateto-high reproducibility and agreement of PD-L1 immune cell expression (using the IC score) in TNBC was found between the SP142, 22C 3 , and 28-8 assays, but the SP263 assay detected higher rates of positive PD-L1 immune cells. However, there was no correlation with ICI therapy response [22]. An exploratory post hoc IMpassion130 substudy investigated 22C3 (CPS) and SP263 (IC score) PD-L1 IHC assays for PD-L1 prevalence, analytical agreement with SP142 (IC score), and clinical value. PD-L1 prevalence was higher using $22 \mathrm{C} 3$ (of note, another score was used) or SP263 compared with SP142. All 3 assays predicted improved progression-free survival in the cohort treated with atezolizumab [43]. Nevertheless, further comparison studies are needed to evaluate the clinical value of the different assays and scores in TNBC.

\section{Issues of PD-L1 Testing}

In brief, we want to highlight some crucial issues of PD-L1 assessment that both clinicians and pathologists should be aware of:

- To date, there is no unique standardized method for PD-L1 evaluation.

- Besides several commercially available CIVD assays with specific defined antibody clones, platforms, and protocols, LDTs are widely used.

- Several scoring methods have been validated in clinical studies.

- Varying antibody clones/assays with different scores are not directly interchangeable.

- For each tumor entity, ICI approval and associated assays and scoring methods differ.

- The approval of available ICI has been linked with specific CIVD assays and scores.

- Therefore, each country/union has different admission requirements depending on the decision of the respective agency evaluating the medicinal products.

- If the approval of each ICI is linked to one specific CIVD and there is no permission to use an LDT, we should be aware that smaller community hospital-associated pathological laboratories, in particular, cannot afford to provide each test for reasons mentioned in the section "PD-L1 IHC Assays and Antibody Clones" [18].

- There are increasing insights into immuno-oncology, PD-L1, and ICI with the frequently changing approval of ICI. Oncologists and pathologists have to update their knowledge and reconsider the procedure of PDL1 assessment regularly.

- Besides annual internal and external quality assessments of the laboratories, pathologists assessing PDL1 IHC should participate in tumor entity-specific PD-L1 evaluation training to improve the interobserver reproducibility.

- For IBC, further clinical studies are necessary to show

- which ICI improve(s) the outcome of breast cancer patients.

- which IBC subtypes (TNBC, HER2+, luminal?) respond to ICI therapy.

- in which setting (neoadjuvant, adjuvant, or metastatic) and in which combination (chemo-/monotherapy?) and sequence ICI should be used.

- which PD-L1 assay and which score predict the response to ICI best.

- what is the extent of tumor heterogeneity, both within a tumor and between a primary tumor and metastasis?

- whether digital image analysis may improve the predictive value of PD-L1 and increase the reproducibility [18].

\section{Conclusion}

Currently, PD-L1 testing is required for adult patients with locally advanced TNBC or mTNBC and intended atezolizumab therapy. In this setting, PD-L1 IHC staining should be performed using an adequate and validated assay. The assessment of PD-L1-positive immune cells using the IC score must be reported by a trained pathologist.

However, since the knowledge about ICI therapies and the clinical value of PD-L1 assays/scores is constantly changing, there should be (i) a continuous update of the level of expertise in this field, and (ii) a lively exchange between oncologists and pathologists to optimize PD-L1 testing for breast cancer patients.

\section{Acknowledgement}

Many thanks to Prof. Wilko Weichert (TUM, Munich, Germany), Mrs. Rudelt, Mrs. Segschneider, and Ms. Landgraf (all FAU, Erlangen, Germany) for technical support. Moreover, we thank all patients who gave their informed consent to participate in all the breast cancer studies mentioned in this review.

\section{Conflict of Interest Statement}

R.E. received honoraria from Roche, Novartis, Pfizer and Eisai, reimbursement of travelling expenses from BioNTech and research funding from NanoString Technologies, Cepheid, BioN- 
Tech and Zytomed Systems. A.H. received honoraria from BristolMyers Sqibb, MSD, Roche, AstraZeneca, Boehringer Ingelheim, Abbvie, Janssen-Cilag and Ipsen and research funding from Cepheid, BioNTech AG, Roche, Janssen-Cilag, NanoString Technologies and AstraZeneca. A.H. also had a consulting/advisory role for Bristol-Myers Sqibb, MSD, Roche, Cepheid, Qiagen, JanssenCilag, AstraZeneca, Ipsen, NanoString Technologies (and an expert testimony), Illumina, 3DHISTECH, Diaceutics.

\section{Funding Sources}

There was no funding for this review.

\section{Author Contributions}

R.E. wrote the manuscript including the literature research. A.H. reviewed the manuscript. The figures were prepared by R.E.

\section{References}

1 Wöckel A, Festl J, Stüber T, Brust K, Stangl S, et al. Interdisciplinary Screening, Diagnosis, Therapy and Follow-up of Breast Cancer. Guideline of the DGGG and the DKG (S3Level, AWMF Registry No. 032/045OL, December 2017) - Part 1 with Recommendations for the Screening, Diagnosis and Therapy of Breast Cancer. Geburtshilfe Frauenheilkd. 2018 Oct;78(10):927-48.

2 WHO. WHO Classification of Tumours. 5th ed. Breast Tumours. Lyon: IARC; 2019. vol. 2, pp. 89-91.

3 Allison KH, Hammond MEH, Dowsett M, McKernin SE, Carey LA, Fitzgibbons PL, et al. Estrogen and Progesterone Receptor Testing in Breast Cancer: ASCO/CAP Guideline Update. J Clin Oncol. 2020;38(12):1346-66.

4 Perou CM, Sørlie T, Eisen MB, van de Rijn M, Jeffrey SS, Rees CA, et al. Molecular portraits of human breast tumours. Nature. 2000 Aug; 406(6797):747-52.

5 Sørlie T, Perou CM, Tibshirani R, Aas T, Geisler S, Johnsen H, et al. Gene expression patterns of breast carcinomas distinguish tumor subclasses with clinical implications. Proc Natl Acad Sci USA. 2001 Sep;98(19): 10869-74.

6 Parker JS, Mullins M, Cheang MC, Leung S, Voduc D, Vickery T, et al. Supervised risk predictor of breast cancer based on intrinsic subtypes. J Clin Oncol. 2009 Mar;27(8):1160-7.

7 Wolff AC, Hammond ME, Allison KH, Harvey $\mathrm{BE}$, Mangu PB, Bartlett JM, et al. Human Epidermal Growth Factor Receptor 2 Testing in Breast Cancer: American Society of Clinical Oncology/College of American Pathologists Clinical Practice Guideline Focused Update. J Clin Oncol. 2018;36(20):2105-22.

8 Lynce F, Shajahan-Haq AN, Swain SM. CDK4/6 inhibitors in breast cancer therapy: current practice and future opportunities. Pharmacol Ther. 2018 Nov; 191:65-73.

9 Pardoll DM. The blockade of immune checkpoints in cancer immunotherapy. Nat Rev Cancer. 2012;12(4):252-64.

10 Brahmer J, Reckamp KL, Baas P, Crinò L, Eberhardt WE, Poddubskaya E, et al. Nivolumab versus Docetaxel in Advanced SquamousCell Non-Small-Cell Lung Cancer. N Engl J Med. 2015 Jul;373(2):123-35.

11 Wilky BA. Immune checkpoint inhibitors: the linchpins of modern immunotherapy. Immunol Rev. 2019 Jul;290(1):6-23.

12 Schmid P, Adams S, Rugo HS, Schneeweiss A, Barrios CH, Iwata H, et al.; IMpassion130 Trial Investigators. Atezolizumab and Nab-Paclitaxel in Advanced Triple-Negative Breast
Cancer. N Engl J Med. 2018 Nov;379(22): 2108-21.

13 Schmid P, Rugo HS, Adams S, Schneeweiss A, Barrios $\mathrm{CH}$, Iwata $\mathrm{H}$, et al. Atezolizumab plus nab-paclitaxel as first-line treatment for unresectable, locally advanced or metastatic triplenegative breast cancer (IMpassion130): updated efficacy results from a randomised, double-blind, placebo-controlled, phase 3 trial. Lancet Oncol. 2020;21(1):44-59.

14 Patel SP, Kurzrock R. PD-L1 Expression as a Predictive Biomarker in Cancer Immunotherapy. Mol Cancer Ther. 2015 Apr;14(4): 847-56.

15 Emens LA, Cruz C, Eder JP, Braiteh F, Chung C, Tolaney SM, et al. Long-term Clinical Outcomes and Biomarker Analyses of Atezolizumab Therapy for Patients with Metastatic Triple-Negative Breast Cancer: A Phase 1 Study. JAMA Oncol. 2019 Jan;5(1):74-82.

16 Vennapusa B, Baker B, Kowanetz M, Boone J, Menzl I, Bruey JM, et al. Development of a PD-L1 Complementary Diagnostic Immunohistochemistry Assay (SP142) for Atezolizumab. Appl Immunohistochem Mol Morphol. 2019 Feb;27(2):92-100.

17 Hanahan D, Weinberg RA. Hallmarks of cancer: the next generation. Cell. 2011 Mar; 144(5):646-74.

18 Gonzalez-Ericsson PI, Stovgaard ES, Sua LF, Reisenbichler E, Kos Z, Carter JM, et al. The path to a better biomarker: application of a risk management framework for the implementation of PD-L1 and TILs as immuno-oncology biomarkers into breast cancer clinical trials and daily practice. J Pathol. 2020 Apr; 250(5):667-84.

19 Scheel AH, Dietel M, Heukamp LC, Johrens K, Kirchner T, Reu S, et al. Harmonized PDL1 immunohistochemistry for pulmonary squamous-cell and adenocarcinomas. Mod Pathol. 2016 Oct;29(10):1165-72.

20 Hirsch FR, McElhinny A, Stanforth D, Ranger-Moore J, Jansson M, Kulangara K, et al. PD-L1 Immunohistochemistry Assays for Lung Cancer: Results from Phase 1 of the Blueprint PD-L1 IHC Assay Comparison Project. J Thorac Oncol. 2017;12(2):208-22.

21 Ma J, Li J, Qian M, Han W, Tian M, Li Z, et al. PD-L1 expression and the prognostic significance in gastric cancer: a retrospective comparison of three PD-L1 antibody clones (SP142, 28-8 and E1L3N). Diagn Pathol. 2018;13(1):91

22 Noske A, Ammann J, Wagner DC, Denkert C, Lebeau A, Sinn P, et al. 359P - Reproducibility and concordance of 4 clinically developed programmed death-ligand 1 (PD-L1) immunohistochemistry (IHC) assays in triple negative breast cancer (TNBC). Ann Oncol. 2019; 30:130-1.

23 Schwamborn K, Ammann JU, Knüchel R, Hartmann A, Baretton G, Lasitschka F, et al. Multicentric analytical comparability study of programmed death-ligand 1 expression on tumor-infiltrating immune cells and tumor cells in urothelial bladder cancer using four clinically developed immunohistochemistry assays. Virchows Arch. 2019;475(5):599-608.

24 [Internet] [cited 24 March 2020]. Available from: https://www.fda.gov/medical-devices/ vitro-diagnostics/list-cleared-or-approvedcompanion-diagnostic-devices-vitro-andimaging-tools

25 Cree IA, Deans Z, Ligtenberg MJ, Normanno N, Edsjö A, Rouleau E, et al.; European Society of Pathology Task Force on Quality Assurance in Molecular Pathology; Royal College of Pathologists. Guidance for laboratories performing molecular pathology for cancer patients. J Clin Pathol. 2014 Nov;67(11):923-31.

26 Cree IA, Booton R, Cane P, Gosney J, Ibrahim M, Kerr K, et al. PD-L1 testing for lung cancer in the UK: recognizing the challenges for implementation. Histopathology. 2016 Aug; 69(2):177-86

27 Eckstein M, Cimadamore A, Hartmann A, Lopez-Beltran A, Cheng L, Scarpelli M, et al. PD-L1 assessment in urothelial carcinoma: a practical approach. Ann Transl Med. 2019 Nov;7(22):690.

28 [Internet] [cited 24 March 2020]. Available from: https://www.pdl1portal.eu/

29 [Internet] [cited 24 March 2020]. Available from: https://www.accessdata.fda.gov/cdrh_ docs/pdf15/P150013S006b.pdf

30 Herbst RS, Baas P, Kim DW, Felip E, PérezGracia JL, Han JY, et al. Pembrolizumab versus docetaxel for previously treated, PD-L1-positive, advanced non-small-cell lung cancer (KEYNOTE-010): a randomised controlled trial. Lancet. 2016 Apr;387(10027): 1540-50.

31 Kulangara K, Hanks DA, Waldroup S, Peltz L, Shah S, Roach C, et al. Development of the combined positive score (CPS) for the evaluation of PD-L1 in solid tumors with the immunohistochemistry assay PD-L1 IHC 22C3 pharmDx. J Clin Oncol. 2017;35(15_ suppl):e14589.

32 [Internet] [cited 24 March 2020]. Available from: https://www.agilent.com/cs/library/ usermanuals/public/29158_pd-11-ihc-22C3pharmdx-nsclc-interpretation-manual.pdf 
33 [Internet] [cited 24 March 2020]. Available from: https://www.accessdata.fda.gov/drugsatfda_docs/label/2019/761034s018lbl.pdf

34 [Internet] [cited 24 March 2020]. Available from: https://www.ema.europa.eu/en/documents/variation-report/tecentriq-h-c004143-x-0017-epar-assessment-report-extension_en.pdf

35 Rugo H, Loi S, Adams S, Schmid P, Schneeweiss A. Carlos H Barrios, et al. Exploratory analytical harmonization of PD-L1 immunohistochemistry assays in advanced triple-negative breast cancer: a retrospective substudy of IMpassion 130 [abstract]. Proceedings of the 2019 San Antonio Breast Cancer Symposium; 2019 Dec 10-14; San Antonio, TX. Cancer Res. 2020;80(4 Suppl):PD1-7.

36 Loibl S, Untch M, Burchardi N, Huober J, Sinn BV, Blohmer JU, et al. A randomised phase II study investigating durvalumab in addition to an anthracycline taxane-based neoadjuvant therapy in early triple-negative breast cancer: clinical results and biomarker analysis of GeparNuevo study. Ann Oncol. 2019 Aug;30(8):1279-88.

37 Schmid P, Cortes J, Pusztai L, McArthur H, Kümmel S, Bergh J, et al.; KEYNOTE-522 Investigators. Pembrolizumab for Early TripleNegative Breast Cancer. N Engl J Med. 2020 Feb;382(9):810-21.
38 Dalenc F, Bachelot T, Fllleron T, et al. Durvalumab compared to maintenance chemotherapy in patients with metastatic breast cancer: results from phase II randomized trial SAFIR02-IMMUNO. San Antonio Breast Cancer Symposium; 2019 Dec 10-14; San Antonio (TX). Abstract GS3-02.

39 VENTANA PD-L1 (SP142) Assay Interpretationshilfe für Urothelkarzinom

40 [Package insert]. VENTANA PD-L1 (SP142) Assay (Article No. 08008540001).

41 Li M, Li A, Zhou S, Xu Y, Xiao Y, Bi R, et al. Heterogeneity of PD-L1 expression in primary tumors and paired lymph node metastases of triple negative breast cancer. BMC Cancer. 2018;18(1):4.

42 Molinero L, Li Y, Chang CW, Maund S, Berg $\mathrm{M}$, Harrison J, et al. Tumor immune microenvironment and genomic evolution in a patient with metastatic triple negative breast cancer and a complete response to atezolizumab. J Immunother Cancer. 2019 Oct;7(1): 274.
43 Rugo HS, Loi S, Adams S. Performance of PDL1 immunohistochemistry (IHC) assays in unresectable locally advanced or metastatic triple-negative breast cancer (mTNBC). Ann Oncol. 2019;30(suppl_5):851-934.

44 [Internet] [cited 24 March 2020]. Available from: https://www.msd.de/fileadmin/ files/fachinformationen/keytruda.pdf

45 [Internet] [cited $24 \mathrm{March}$ 2020]. Available from: https://fi.b-ms.de/Opdivo

46 [Internet] [cited 24 March 2020]. Available from: https://www.agilent.com/cs/library/ packageinsert/public/P03914EFG_05.pdf

47 [Internet] [cited 24 March 2020]. Available from: https://www.ema.europa.eu/en/ documents/product-information/tecentriqepar-product-information_en.pdf

48 [Internet] [cited 24 March 2020]. Available from: http://reagent-catalog.roche.com/ product $/ 1827$ ? type $=2357$

49 [Internet] [cited 24 March 2020]. Available from: https://diagnostics.roche.com/ch/ de/products/tests/ventana-pd-11-_sp263-assay2.html\#productInfo

50 [Internet] [cited 24 March 2020]. Available from: https://www.ema.europa.eu/en/documents/product-information/imfizi-eparproduct-information_en.pdf 